\title{
Universidad Tecnológica de Tula-Tepeji
}

\author{
Sandra López Acosta, ${ }^{1}$ Víctor Alfredo Nolasco Arizmendi ${ }^{2}$
}

RESUMEN: El Programa Educativo de Nanotecnología de la Universidad Tecnológica de Tula-Tepeji (UTTT) forma, en seis cuatrimestres, técnicos superiores universitarios, siguiendo cuatro ejes pedagógicos. El último cuatrimestre del programa es una estadía en el sector productivo para desarrollar un proyecto que atienda una necesidad real aplicando los conocimientos que adquirieron en el programa. Los estudiantes reciben una formación que los convierte en profesionistas integrales con conocimientos y destrezas para producir y caracterizar materiales nanotecnológicos, facilitando su ingreso al mercado laboral. El programa tiene relación con empresas, universidades y centros de investigación para estadías de los estudiantes e infraestructura que incluye instalaciones y equipo básico y avanzado. Los profesores del programa hacen investigación y desarrollos tecnológicos en el área de biotecnología y nanotecnología ambiental con colaboración académica interna y con otras instituciones del país. Además, el programa participa en actividades de difusión de la ciencia y la nanotecnología. Con el Programa Educativo de Nanotecnología, la UTTT participa en un área estratégica y de alta innovación para el país.

PALABRAS CLAVE: Nanotecnología, técnico superior universitario, universidades tecnológicas.

ABSTRACT: The Nanotechnology Educational Program of the Technological University of TulaTepeji (UTTT) forms, in six quarters, university technical technicians following four pedagogical axes. The last quarter of the program is a stay in the productive sector developing a project that meets a real need applying the knowledge acquired in the program. The education that the students receive in the UTTT results in professionals with integral training and knowledge and skills to produce and characterize nanotechnological materials, which facilitates their entry into the labor market. The program is supported by companies, universities and research centers for student stays. The program infrastructure includes basic and advanced facilities. The professors do research and technological developments in the area of biotechnology and environmental nanotechnology. They have academic collaboration with other institutions of the country. In addition, all the faculty participates in activities to spread science and nanotechnology topics. With the Educational Program of Nanotechnology, UTTT participates in a strategic and high innovation area for the country.

KEYWORDS: Nanotechnology, university technical technicians, technological universities.

Recibido: 5 de julio de 2016 Aceptado: 7 de noviembre de 2016.

1 Directora del Programa Educativo de Nanotecnología en la Universidad Tecnológica de Tula-Tepeji, (UTTT). Av. Universidad Tecnológica No. 1000 Col. El 61, El Carmen, Tula de Allende, Hgo. C.P. 42830 Tel. (773) 732-9113. Correspondencia: (ambiental@uttt.edu.mx).

2 Profesor de Tiempo Completo del Programa Educativo de Nanotecnología en la UTTT. Tel. (773) 732-9100 ext. 475. Correspondencia: (vnolasco@uttt.edu.mx). 


\section{Antecedentes de las universidades tecnológicas en México}

El Subsistema de Universidades Tecnológicas en nuestro país nace a principios de la década de los noventa, con la finalidad de ampliar y diversificar la oferta educativa superior al crear una modalidad de estudios correspondiente al nivel 5B (de acuerdo con la Clasificación Internacional Normalizada de la Educación, CINE), en las áreas del conocimiento tecnológico-administrativas. Esta opción educativa brinda a los egresados de bachillerato dos años de estudios intensivos en los que desarrollan destrezas específicas orientadas hacia la práctica que facilitan su ingreso al mercado laboral. Es con estos precedentes que surge el técnico superior universitario (TSU).

El modelo educativo de las universidades tecnológicas está basado en 6 ejes: calidad, pertinencia, intensidad, flexibilidad, polivalencia y continuidad; atributos característicos de todos los programas educativos, que impactan en el desarrollo económico y social de sus regiones. Al mismo tiempo, se privilegia la formación integral de los estudiantes, dotándolos de una educación tecnológica especializada pero también de las herramientas necesarias que los lleven a formarse en lo humano y lo social. Es importante resaltar que las universidades tecnológicas se ubican mayormente en localidades en las que no existía alguna opción de educación superior; así, uno de sus objetivos es brindar una formación profesional a los jóvenes que conforman la zona de influencia de cada UT, de manera que pueden insertarse en el mercado laboral y contribuir al desarrollo económico de sus comunidades. Por tal motivo el proceso de ingreso facilita el acceso para aquellos aspirantes que han concluido su bachillerato y cuentan con un perfil académico acorde con el programa educativo al que desean incorporarse.

En 2009, el Subsistema se planteó la necesidad de atender la demanda de sus egresados quienes pedían la continuidad de su preparación para obtener un título como ingenieros. Fue entonces cuando se realizaron los estudios de factibilidad correspondientes, en donde el sector empresarial tuvo un papel decisivo en el diseño de los planes de estudio de los programas que se ofertarían como segundo ciclo o ingeniería. Esta opción provee a los técnicos superiores universitarios la posibilidad de continuar sus estudios por 1 año 8 meses y obtener un título como ingeniero en el área de su especialidad.

De este modo, las universidades tecnológicas ofrecen en un periodo de 3 años 8 meses, la obtención de dos títulos profesionales y una amplia experiencia en el sector laboral, lo cual representa una ventaja competitiva para los egresados de estas instituciones educativas.

A la fecha, existen 103 universidades tecnológicas distribuidas en 31 estados de la República que ofrecen programas de técnico superior universitario y programas de ingeniería. De ellos, más de 200 programas se encuentran en el nivel 1 de la evaluación que otorgan los Comités Interinstitucionales para la Evaluación de la Educación Superior (CIEES), o cuentan con 
acreditaciones ante organismos reconocidos por el Consejo para la Acreditación de la Educación Superior (COPAES), además de tener implantados sistemas de gestión de calidad certificados en ISO-9000.

\section{Universidad Tecnológica de Tula-Tepeji}

Enclavada al suroeste del estado de Hidalgo, entre los municipios que le dan su nombre, la Universidad Tecnológica de Tula-Tepeji tiene el privilegio de ser una de las 3 primeras universidades tecnológicas en el país. Comenzó sus operaciones en septiembre de 1991 con 2 programas educativos de técnico superior universitario. Actualmente, en la UTTT se imparten 17 programas educativos de TSU, 8 ingenierías y 4 ingenierías técnicas; distribuidas entre el campus central y dos unidades académicas localizadas en los municipios de Chapulhuacán y Tepetitlán. Lo anterior ha permitido que la institución sea reconocida como un referente de educación superior en la región.

El trabajo llevado a cabo en la UTTT se sustenta en la calidad del personal académico y administrativo que labora en ella y su capacitación permanente en instituciones altamente reconocidas tanto nacionales como del extranjero. Hoy en día, cuenta con 89 profesores de tiempo completo, los cuales ejercen funciones de docencia, vinculación y difusión. De ellos, 52 cultivan líneas de investigación dentro de los 13 cuerpos académicos de la institución o participan en proyectos de desarrollo tecnológico con las empresas. Los profesores por asignatura o de tiempo parcial son profesionistas que trabajan en el sector productivo en áreas afines a las asignaturas que imparten.

Los programas educativos que se ofrecen en la Universidad responden a las necesidades de la zona de influencia, pues antes de abrir una nueva carrera, se analiza la demanda laboral y la oferta educativa en el entorno y, con los resultados de esta exploración, se define la pertinencia de su apertura. En este proceso, los empresarios tienen un papel fundamental para la definición de los contenidos académicos con el objetivo de que sean acordes a sus requerimientos de personal.

La población estudiantil procede en su mayoría de las localidades cercanas a Tula en Hidalgo y norte del Estado de México, principalmente. Alrededor de un $4 \%$ de la matrícula total de la institución proviene de comunidades indígenas, y cerca del $10 \%$ se identifican como estudiantes en condiciones de vulnerabilidad, debido a que trabajan y estudian, son padres o madres solteros(as), tienen alguna enfermedad que les impide asistir a clases de manera regular o bien presentan alguna discapacidad. Es importante señalar que un alto porcentaje de la matrícula proviene de hogares de escasos recursos económicos y en ocasiones, representan el primer integrante de sus familias en acceder a la educación superior. Éste es el contexto en el que se desarrollan nuestros estudiantes, de ahí que los apoyos ofrecidos por la UTTT se encaminan a afianzar su permanencia dentro de la institución, de manera que puedan concluir su carrera profesional e incorporarse al sector productivo. 


\section{Programa Educativo de Técnico Superior Universitario en Nanotecnología}

La creación del Programa Educativo de Técnico Superior Universitario en Nanotecnología surge como una iniciativa de la Coordinación General de Universidades Tecnológicas y Politécnicas, para atender áreas estratégicas mediante la formación de profesionistas con capacidades para el desarrollo de investigación y un sólido abordaje interdisciplinario en las ciencias básicas y aplicadas.

En la UTTT el Programa de TSU en Nanotecnología se inicia en septiembre de 2011, con una matrícula de 14 estudiantes. Debido a su nueva creación, el programa se fortaleció gracias a la vinculación con otras instituciones de educación superior y centros de investigación que complementan la formación profesional con los conocimientos prácticos que se requieren. Desde entonces y de manera periódica, los estudiantes realizan visitas al Centro de Química Sustentable de la UAEMEX y al Centro de Micro y Nanotecnologías del IPN para conocer la operación de microscopios electrónicos; al Centro de Desarrollo Industrial (CIDESI), para ampliar sus conocimientos sobre tratamientos térmicos que se dan a ciertos materiales; al Instituto de Ingeniería de la UNAM, para caracterizar los materiales nanoestructurados obtenidos en los laboratorios de la UTTT; a la empresa Termoinnova, para operar el equipo de difracción de rayos $\mathrm{X}$ que se encuentra en comodato dentro de la misma. Esto por mencionar algunas de las colaboraciones que mantiene la institución. A la fecha, se han formado 4 generaciones con un total de 22 egresados. De ellos, 2 están cursando estudios de maestría en el área de materiales; 19, se encuentran estudiando ingeniería en nanotecnología en alguna otra UT del Subsistema y 1 obtuvo una beca para cursar estudios sobre polímeros en Francia por un año. Quienes continuaron estudiando, combinan el estudio con el trabajo en Centros de Investigación, principalmente en el estado de Querétaro (CINVESTAV).

Las asignaturas que conforman el plan de estudios están organizadas en cuatro ejes pedagógicos que incluyen ciencias básicas aplicadas, formación tecnológica, lenguas y métodos y habilidades gerenciales. Todas ellas abonan a la formación de un profesionista los conocimientos y destrezas necesarios para: 1) producir materiales nanotecnológicos, mediante procedimientos de síntesis e incorporación de nanomateriales establecidos, y, 2) evaluar materiales nanoestructurados a través de técnicas de caracterización para determinar sus propiedades físicas y químicas; que constituyen las dos competencias específicas del perfil de egreso.

El programa académico está distribuido en seis cuatrimestres, el último de los cuales, denominado estadía, se realiza en sector productivo desarrollando un proyecto que atienda una necesidad real mediante la aplicación de los conocimientos adquiridos durante la carrera. Las estadías de los estudiantes de nanotecnología se han llevado a cabo en organismos diversos como el 
Centro de Nanociencias y Nanotecnología, en Ensenada; el Centro de Investigaciones en Materiales Avanzados, en Chihuahua; el Instituto Potosino de Investigación en Ciencia y Tecnología, en San Luis Potosí; el Centro de Nanociencias y Micro y Nanotecnologías, de la Ciudad de México; la empresa Termoinnova, en Tula, Hidalgo, y el Instituto de Ingeniería de la UNAM.

Los temas abordados en los reportes de estadía incluyen:

- Preparación y caracterización de películas delgadas de nanocúmulos de silicio embebidos en nitruro de silicio depositadas por plasma, por el método de rocío pirolítico.

- Estabilidad de AgNPs y Zeo-AgNPs bajo diferentes condiciones ambientales.

- Síntesis asistida por microondas de nanoestructuras.

- Cinética en la electrocoagulación y floculación de nanopartículas de plata obtenidas por biorreducción.

- Síntesis y caracterización magneto-estructural de aleaciones magnéticas, de cintas micro y nanocristalinas de aleaciones Ni50Mn35In15 tipo Heusler, de películas de aleaciones Mn5Ge3 dopadas con carbono.

- Evaluación de fotocatalizadores nanoestructurados para la descomposición de la molécula del agua.

- Síntesis de hidroxiapatitas nanométricas.

- Estudio de la topología de superficies en piezas de acero.

- Tratamiento termoquímico producido sobre una superficie de acero.

- Uso de nanomateriales fotocatalíticos para la producción de hidrógeno.

- Investigación de propiedades catalíticas y fisicoquímicas de nanopartículas metálicas.

Durante su estancia en la Universidad, los estudiantes tienen la oportunidad de asistir a congresos que enriquecen su formación académica. De este modo, se participa anualmente en el Simposio de Divulgación en Nanociencias y Nanotecnología (Nanocytec), reunión organizada por las universidades tecnológicas del país que imparten nanotecnología, en donde se presentan los trabajos realizados por docentes y estudiantes. En la primera edición de este evento, alumnos de la Universidad Tecnológica de Tula-Tepeji obtuvieron el primer lugar en el concurso de carteles con el trabajo denominado "Síntesis de nanopartículas de hematita $\mathrm{A}-\mathrm{Fe}_{2} \mathrm{O}_{3}$ utilizando el método de precipitación controlada". Asimismo, asisten al Foro Internacional Nano Monterrey, donde tienen la oportunidad de establecer contacto con empresas en las que podrían realizar su estadía, además de introducirse en los aspectos de vanguardia relacionados con su carrera. En estos eventos, los estudiantes presentan trabajos de investigación en los que colaboran con sus profesores dentro de la Universidad.

De igual manera, los estudiantes participan en la convocatoria denominada 
"Ensayo del Agua", emitida anualmente por la American Water Works Association (AWWA) - Capítulo México. En su 6a edición, una estudiante de la $3^{\mathrm{a}}$ generación de TSU en Nanotecnología obtuvo el tercer lugar nacional con el tema: "Recreando el agua con la ciencia de los nanómetros, ipara beber, para vivir!".

\section{Infraestructura}

El programa educativo cuenta con laboratorios de química básica, analítica y microbiología, dedicados a la docencia, cuyo equipamiento consiste de balanzas analíticas, campanas de extracción y de flujo laminar, medidores de $\mathrm{pH}$ y conductividad, rotavapor, parrillas con agitación, refrigeradores, estufas bacteriológicas, muflas, autoclaves, termobalanza, baño ultrasónico, microscopios ópticos, centrífuga, incubadora y material de cristalería. También cuenta con un laboratorio de instrumentación analítica que alberga los equipos de espectroscopía UV-VIS, infrarroja y de absorción atómica, así como un horno digestor. Entre ambos laboratorios, hay un espacio en el que se concentra el equipo a escala piloto, tal como la unidad de generación eléctrica, unidad de destilación, reactor químico, banco de motores y las plantas de tratamiento de aguas por filtración sobre arena, ultrafiltración, ósmosis inversa, tratamiento aerobio, tratamiento anaerobio, floculación-coagulación, intercambio iónico y filtración sobre soporte. Este equipamiento, brinda soporte a la parte práctica de los diferentes programas educativos del Área de Química y Nanotecnología que oferta la Institución.

\section{Investigación y proyectos de desarrollo tecnológico}

Los profesores de tiempo completo que participan en el programa cultivan la línea de investigación de biotecnología y nanotecnología ambiental, dentro del Cuerpo Académico de Ingeniería y Sistemas Ambientales, el cual se encuentra en consolidación ante el Programa para el Desarrollo Profesional Docente (PRODEP). Algunos académicos pertenecen al Sistema Nacional de Investigadores (SNI).

Dentro de los proyectos de investigación realizados, se pueden mencionar los siguientes:

- Síntesis, inhibición microbiológica y tratamiento del agua residual de materiales nanoestructurados de plata de tamaño controlado obtenidos por técnicas de biorreducción con un extracto de Tillandsia Recurvata L. proveniente de la región de Tula, Hidalgo. (El cual obtuvo financiamiento por parte de PRODEP).

- Remoción biológica de Fe y Mn de agua subterránea mediante la obtención de óxidos biogénicos con potencial valor agregado. (Financiado por PRODEP). 
Por otra parte, se han realizado proyectos de desarrollo tecnológico en apoyo a las empresas de la región. Algunos de éstos son:

- Incorporación de nanomateriales de origen natural a matrices poliméricas para la generación de productos de alta resistencia. (Financiado por CONACyT-PEI 2014).

- Generación de nanocompuestos poliméricos a partir de polímeros reciclados y desarrollo de componentes multicapa mediante coextrusión y soplado con propiedades de descarga electrostática "nanoplastics"/estudio para desarrollar un compuesto polimérico. (Financiado por CONACyT-PEI 2015).

\section{Colaboraciones}

El Cuerpo Académico de Ingeniería y Sistemas Ambientales participa con diversas IES en el desarrollo de investigaciones conjuntas. Así, forma parte de la Red Temática de Colaboración Académica en Ingeniería de Procesos Avanzados de Sistemas Ambientales, integrada por la Universidad Autónoma del Estado de Hidalgo, la Universidad Michoacana de San Nicolás Hidalgo, la Universidad Politécnica de Pachuca y la Universidad Tecnológica de Tula-Tepeji.

Actualmente, se participa con la Universidad Tecnológica de Ciudad Juárez en el desarrollo de investigaciones sobre el uso de la nanotecnología aplicada al tratamiento de aguas.

\section{Actividades de difusión y divulgación de la ciencia}

La UTTT ha realizado actividades de difusión y divulgación de la nanociencia y nanotecnología, como:

1. Participar en la traducción español-hñä-hñu del texto Vamos a conocer la nanotecnología, del Dr. Noboru Takeuchi, investigador del Centro de Nanociencias y Nanotecnología, coeditada por la Universidad Nacional Autónoma de México y la Universidad Tecnológica de Tula-Tepeji. Lo anterior, con el objetivo de acercar y divulgar la ciencia entre los niños y jóvenes de las comunidades indígenas, además de apoyar la conservación y revitalización de las lenguas indígenas de México. La publicación explica de manera amena y con coloridas ilustraciones, diversos aspectos de la nanotecnología; incluye un disco compacto donde se pueden escuchar en español y hñä-hñu los textos del libro. La traducción estuvo a cargo de Adela Calva Reyes y Diana Ángeles Camacho, integrantes de la comunidad de San Ildefonso, municipio de Tepeji del Río, Hgo.

2. En junio de 2014, la Universidad fue sede del International Multidisciplinary Join Meeting, evento en el que se mostraron los avances sobre nuevos 
procesos para la obtención de materiales nanoestructurados, y donde se analizó el impacto de la $\mathrm{NyN}$ en diversos campos como la medicina, biotecnología, agroindustria, así como sus implicaciones económicas, sociales y ambientales, entre otras. La reunión multidisciplinaria se realizó en la ciudad de Pachuca, del 9 al 13 de junio de 2014. El International Multidisciplinary Join Meeting congregó dos de las reuniones más importantes en el área de la NyN: el International Topical Meeting on Nanostructured Materials and Nanotechnology (Nanotech), y, el Encuentro Internacional e Interdisciplinario en Nanociencia y Nanotecnología (Nanomex). Los eventos se llevaron a cabo gracias a la colaboración con la Universidad Nacional Autónoma de México y sus diferentes centros de investigación: Centro de Nanociencias y Nanotecnología, Centro de Investigaciones Interdisciplinarias en Ciencias y Humanidades y el Centro de Ciencias Aplicadas y Desarrollo Tecnológico.

A continuación las referencias de los trabajos realizados por los docentes y estudiantes del programa educativo, a la fecha:

a) "Una alternativa para la remoción de nanopartículas de plata coloidales de agua por un tratamiento de electrocoagulación". Autores: Víctor Alfredo Nolasco Arizmendi, Abygail Adarely Martínez Mendoza, Haly Surisadday Juárez Pérez.

Evento: Factor Sinergia - Congreso Internacional de Investigación Aplicada e Innovación Tecnológica 2015. 25 al 27 de noviembre. San Juan del Río, Qro., Méx.

b) "Síntesis verde y caracterización de materiales compuestos de plata utilizando extractos acuosos". (Póster).

Autores: Víctor Alfredo Nolasco Arizmendi. UTTT.

Evento: VII Congreso Nacional de Ciencia e Ingeniería en Materiales.

c) "Tratamiento de nanopartículas de plata por técnicas de precipitación”.

Autor: Nolasco Arizmendi Víctor Alfredo, UTTT.

Evento: IV Congreso de Nanociencias y Tecnología 2015.

d) "Electrocoagulation system used as an electrochemical methodology to obtain Zn nanostructures of wastewater".

Autores: Víctor Nolasco Arizmendi, Lizeth Janeth Beltrán Ontiveros, María del Rosario Valencia Romero, Karla Edith Vega Chavez, Maricruz Rocha Rubio, Carlos Iván Rodríguez Rodríguez.

Evento: IX International Conference on Surfaces, Materials and Vacuum. 
e) "Nanotecnología como un método para purificar el agua". Autores: María Fernanda Campuzano y Ricardo Larios, estudiantes UTTT, coinciden en señalar que es importante encontrar métodos para reutilizar el agua. Disponible en : <http://www.oem.com.mx/ elsoldezamora/notas/n4161909.htm $>$.

f) "Efecto en el plasmón de resonacia superficial (SPR) de nanopartículas de plata obtenidas por biorreducción con Citrus lemon L. con diferentes estados de madurez".

Autores: Nolasco-Arizmendi V.A., Martínez-Mendoza A.A., Martínez-Muñoz P.E., Rodríguez- Rodríguez C. I.

Evento: Food \& Biotechnology International Congress 2016.

ISBN de las memorias: 978-607-96797-2-9.

g) "Remoción y reducción de iones Fe utilizando como floculante un extracto de Solanum tuberosum L.".

Autores: Nolasco-Arizmendi V.A., Martínez-Muñoz P. E., Rodríguez Rodríguez C. I.

Evento: Food \& Biotechnology International Congress 2016.

ISBN de las memorias: 978-607-96797-2-9.

\section{Participaciones en eventos}

En abril del presente año, se asistió al workshop "Interdisciplina aplicada y tecnologías disruptivas para Latinoamérica”, organizado por el Centro Latinoamericano de Formación Interdisciplinaria (CELFI) del Ministerio de Ciencia, Tecnología e Innovación Productiva de la República Argentina. Allí, diversos especialistas expusieron sus trabajos sobre el impacto de las nuevas tecnologías disruptivas, entre las que se encuentra la nanotecnología, y, la democratización de conocimientos. En esta ocasión, el CELFI financió la participación de 25 becarios de diversos países de Latinoamérica, entre los cuales fue beneficiado un docente de la Universidad Tecnológica de Tula-Tepeji, así como otros participantes de países como Brasil, Colombia, Venezuela y Ecuador. El trabajo presentado por la representación de la UTTT es un estudio sobre la estabilidad de nanopartículas de plata en un proceso de floculación.

\section{Perspectivas}

El plan de desarrollo del Programa Educativo de Nanotecnología incluye su acreditación, en el corto plazo, ante los organismos del COPAES. Por otra parte, a partir de 2017, los estudiantes recibirán una certificación en el estándar "Análisis de materiales mediante técnicas cualitativas", como parte de su formación profesional. Esto alineado a la entidad de certificación del CONOCER con la que cuenta la Universidad. 
En materia de investigación, en 2017 el Cuerpo Académico de la carrera se someterá a un proceso de evaluación ante el PRODEP. Se espera obtener el nivel de Cuerpo Académico Consolidado. 